\title{
Frequency and Clinical Impact of Serious Adverse Events on Post-Stroke Recovery with NeuroAiD (MLC601) versus Placebo: The CHInese Medicine Neuroaid Efficacy on Stroke Recovery Study
}

\author{
Narayanaswamy Venketasubramanian ${ }^{a}$ Rajesh B. Moorakonda ${ }^{b}$ Qingshu Lu ${ }^{b}$ \\ Christopher L.H. Chen ${ }^{c}$ On behalf of the CHIMES Investigators \\ ${ }^{a}$ Raffles Neuroscience Centre, Raffles Hospital, Singapore, Singapore; b Singapore Clinical Research Institute, \\ Singapore, Singapore; ' Department of Pharmacology, National University of Singapore, Clinical Research Centre, \\ Singapore, Singapore
}

\section{Keywords}

Clinical trial · NeuroAiD · MLC601 - Serious adverse events ·

Clinical impact $\cdot$ Stroke

\begin{abstract}
Background: Most comparative clinical trials are designed to assess the treatment effect for efficacy endpoints, with less emphasis on the analysis of safety outcomes. However, an extensive analysis of safety data could demonstrate beneficial results in terms of effectiveness by reducing serious adverse events (SAEs), and their unfavourable clinical impact on the study population. We aimed to conduct an exploratory analysis of the CHInese Medicine Neuroaid Efficacy on Stroke recovery (CHIMES) study safety database comparing the frequency of SAEs and their clinical impacts among subjects having received MLC601 or placebo during the first 3 months post-stroke. Methods: Analyses were performed by using the safety database of the multicentre, randomised, double-blind, placebo-controlled CHIMES study of 3 months of NeuroAiD versus placebo in subjects with acute ischaemic stroke of intermediate severity in the preceding $72 \mathrm{~h}$. SAEs as reported by investigators at any time-point during the
\end{abstract}

3-month study were analysed on their frequency and that of any of their outcomes (death, and life threatening, new and/ or prolonged hospitalisation, disability, and medical importance, in surviving subjects), as well as their time to onset and resolution. Results: Of the 1,099 subjects in the CHIMES study, 1,087 were included in the safety analysis (MLC601 = 542) and (placebo $=545$ ); the 12 who did not receive study treatment were excluded. There was a total of 135 subjects with SAEs $(M L C 601=60$, placebo $=75)$. At baseline, overall, subjects with SAEs were older and had lower MMSE score. In the MLC601 group, they had higher NIHSS score, and more frequently a history of ischaemic heart disease and hyperlipidaemia. The number of SAEs per subjects was statistically significantly lower in the MLC601 group than placebo one, especially for subjects with $\geq 2$ SAEs (6.7 vs. $29.3 \%$; $p<0.001)$. This benefit was seen throughout the study period and during the initial hospitalisation. The main clinical impact of SAEs was an increase in hospitalisation time, reduced in the MLC601 arm with the rate of subjects hospitalised for a prolonged period being significantly threefold lower in surviving subjects ( 1.1 vs. $3.7 \% ; p<0.01)$. Conclusions: This post hoc analysis of SAEs from the CHIMES study database shows that subjects receiving a 3-month course of MLC601 experi-

\begin{tabular}{ll}
\hline KARGER & ( 2020 The Author(s) Karger \\
& Published by S. Karger AG, Basel Open ciccess \\
karger@karger.com & This article is licensed under the Creative Commons Attribution- \\
www.karger.com/ced & NonCommercial-NoDerivatives 4.0 International License (CC BY- \\
& NC-ND) (http://www.karger.com/Services/OpenAccessLicense). \\
Usage and distribution for commercial purposes as well as any dis- \\
tribution of modified material requires written permission.
\end{tabular}

Narayanaswamy Venketasubramanian, FRCP

Raffles Neuroscience Centre

585 North Bridge Road

\#02-00 Raffles Hospital, Singapore 188770 (Singapore)

E-Mail drnvramani@gmail.com 
enced fewer SAEs, with lower rates of harmful clinical impacts, especially in terms of hospitalisation duration. These findings could translate to a benefit in terms of reduction of both healthcare burden and additional medical costs.

(c) 2020 The Author(s)

Published by S. Karger AG, Basel

\section{Introduction}

"Primum non nocere" is a basic medical principle, originally ascribed to Hippocrates, meaning "First, do no harm" [1]. This principle should guide our therapeutic decisions for our patients, knowing that fine tuning will be necessary to aim at the best benefit-risk ratio according to disease severity, patient's clinical status, expected efficacy and potential side effects. Safety analysis plays a key role in the regulatory process of drug approval $[2,3]$, and the benefit assessment of drugs after approval [4]. Therefore, an important part of the clinical development of new treatments must focus on the collection and analysis of adverse events (AE), especially serious AEs ones (SAE).

Investigators and drug developers must actively guard against consequences of any philosophical dichotomy between safety and efficacy. Suboptimal efficacy can have serious adverse consequences, such as prolonged hospitalisation, complications and even death, with associated cost increases [5]. Nevertheless, usually comparative clinical trials are most designed by calculating study power and sample size mainly for efficacy purpose. Thus, randomised controlled trials are often underpowered to detect some treatment-related side effects, let alone to analyse their consequences and clinical impacts, the information provided in many publications being limited to the list of most frequent AEs during the study period [6].

A thorough analysis of safety data could demonstrate beneficial results in terms of effectiveness by reducing SAE frequency, and their unfavourable clinical impact on study population. To avoid any dichotomy between safety and efficacy, it has been recommended to analyse adverse medical events as a whole set, where efficacy and safety overlap [5]. The rationale is that fewer SAEs drives better safety and improves the treatment effect by limiting additional hurdles to obtain maximum benefit. As an example of this kind of safety/efficacy analysis, a recent retrospective study reported that mechanical thrombectomy appears to be safe and effective in anticoagulated patients, ineligible for thrombolysis, by achieving haem- orrhage rates similar to those of patients not on anticoagulant therapy [7]. In the CHInese Medicine Neuroaid Efficacy on Stroke recovery (CHIMES) study conducted in subjects having experienced an ischaemic stroke in the previous $72 \mathrm{~h}$, we observed at 3 months a lower frequency of SAEs with MLC601 (12\%) vs. placebo (18\%) [8], as well as halving of vascular events [9]. Based on these previous positive outcomes, we aimed at conducting an extensive exploratory analysis of the CHIMES study safety database. Our objective was to test the hypothesis that by reducing SAE frequency and their clinical impacts during the post-stroke recovery phase, MLC601 compared to placebo could increase the overall benefit for patients after stroke both in terms of efficacy and safety.

\section{Methods}

Study Design and Population

As previously published [10], the CHIMES study is a multicentre, randomized, double-blinded, placebo-controlled trial comparing a 3-month course of MLC601 with placebo in subjects with acute ischaemic stroke of intermediate severity in the preceding $72 \mathrm{~h}$. Subjects were randomized to receive either MLC601 or matching placebo at a dose of 4 capsules 3 times daily for 3 months. Each 400 mg MLC601 capsule contained extracts from 9 herbal components (Radix astragali, Radix salviae mitorrhizae, Radix paeoniae rubra, Rhizoma chuanxiong, Radix angelicae sinensis, Carthamus tinctorius, Prunus persica, Radix polygalae and Rhizoma acori tatarinowii) and 5 non-herbal components (Hirudo, Eupolyphaga seu steleophaga, Calculus bovis artifactus, Buthus martensii and Cornu saigae tataricae). Study treatment was added on to standard stroke care including antiplatelet therapy, control of vascular risk factors, and appropriate rehabilitation. Any SAE occurring during the 3-month study period was recorded, with data regarding diagnosis of the event, date of onset/resolution, severity, action taken concerning study treatment, relatedness to study drug, treatment given, seriousness criteria, causality, expectedness, and outcome. All SAE were adjudicated by a committee blinded to study arm allocation.

\section{Study Objectives}

One of the objectives was to identify risk factors for SAEs. The primary objective of this analysis was to compare between treatment arms, as a safety analysis, the number of SAEs in the whole study population and in subsets of subjects with SAEs. Another main objective was to compare, as a secondary efficacy analysis, the clinical impacts of SAEs between treatment arms with respect to death, new and/or prolonged hospitalisation, life-threatening event, disability, and important medical event (IME). SAEs were classified as IME by 2 approaches: (a) by investigators at study sites based on their medical judgement; (b) according to the Medical Dictionary for Regulatory Activities (MedDRA) term list version 22.1 of European Medicines Agency, which was designed for sharing regulatory information about human medical products [11]. 
Table 1. Baseline characteristics of subjects with SAEs compared to those without SAE in both treatment arms

\begin{tabular}{|c|c|c|c|c|c|c|}
\hline \multirow[t]{2}{*}{ Characteristics } & \multicolumn{3}{|l|}{ MLC601 } & \multicolumn{3}{|l|}{ Placebo } \\
\hline & $\begin{array}{l}\text { with SAE } \\
(n=60)\end{array}$ & $\begin{array}{l}\text { without SAE } \\
(n=482)\end{array}$ & $\begin{array}{l}\text { level of } \\
\text { significance }\end{array}$ & $\begin{array}{l}\text { with SAE } \\
(n=75)\end{array}$ & $\begin{array}{l}\text { without SAE } \\
(n=470)\end{array}$ & $\begin{array}{l}\text { level of } \\
\text { significance }\end{array}$ \\
\hline Gender, female, $n(\%)$ & $20(33.3)$ & $188(39.0)$ & & $23(30.7)$ & $169(36.0)$ & \\
\hline Age, years, mean (SD) & $65.0(11.2)$ & $60.9(10.6)$ & $* *$ & $65.6(10.8)$ & $60.9(11.8)$ & $* *$ \\
\hline BMI, $\mathrm{kg} / \mathrm{m}^{2}$, mean $(\mathrm{SD})$ & $24.3(4.0)$ & $24.5(3.9)$ & & $24.9(4.9)$ & $24.5(3.8)$ & \\
\hline OTT $\geq 48$ h, $n(\%)$ & $38(63.3)$ & $314(65.1)$ & & $47(62.7)$ & $294(62.6)$ & \\
\hline NIHSS, mean (SD) & $9.7(3.3)$ & $8.6(2.4)$ & $*$ & $8.8(2.9)$ & $8.5(2.5)$ & \\
\hline NIHSS score $\geq 10, n(\%)$ & $27(45.0)$ & $162(33.6)$ & & $24(32.0)$ & $144(30.6)$ & \\
\hline MMSE, mean (SD) & $19.0(9.6)$ & $24.7(6.6)$ & $* * *$ & $22.2(8.5)$ & $24.9(6.3)$ & $*$ \\
\hline \multicolumn{7}{|l|}{ Pre-stroke mRS, $n(\%)$} \\
\hline Previous myocardial infarction & $5(8.3)$ & $9(1.9)$ & & $6(8.0)$ & $14(3.0)$ & \\
\hline Angina & $4(6.7)$ & $9(1.9)$ & & $3(4.0)$ & $20(4.3)$ & \\
\hline Hypertension & $52(86.7)$ & $389(80.7)$ & & $60(80.0)$ & $381(81.1)$ & \\
\hline Diabetes mellitus & $23(38.3)$ & $146(30.3)$ & & $34(45.3)$ & $145(30.9)$ & $*$ \\
\hline Hyperlipidaemia & $39(65.0)$ & $222(46.1)$ & $* *$ & $51(68.0)$ & $216(46.0)$ & $* * *$ \\
\hline $\mathrm{BMI} \geq 30$ & $6(10.0)$ & $31(6.4)$ & & $10(13.3)$ & $37(7.9)$ & \\
\hline Peripheral vascular disease & 0 & $5(1.0)$ & & 0 & $3(0.6)$ & \\
\hline Smoking & $35(58.3)$ & $214(44.4)$ & $*$ & $36(48.0)$ & $210(44.7)$ & \\
\hline Alcohol & $19(31.7)$ & $136(28.2)$ & & $19(25.3)$ & $138(29.4)$ & \\
\hline Previous ischaemic stroke & $7(11.7)$ & $42(8.7)$ & & $7(9.3)$ & $42(8.9)$ & \\
\hline Previous haemorrhage stroke & $1(1.7)$ & $4(0.8)$ & & $1(1.3)$ & $2(0.4)$ & \\
\hline TIA & $2(3.3)$ & $14(2.9)$ & & $2(2.7)$ & $11(2.3)$ & \\
\hline
\end{tabular}

$\S$ Level of significance for comparisons between with SAE versus without SAE subjects in each treatment arms: ${ }^{*} p<0.05 ; * * p<0.01$, *** $p<0.001$, and blank indicates $p>0.05$.

SAE, serious adverse event; BMI, body mass index; OTR, time from stroke onset to randomization; OTT, time from stroke onset to treatment; NIHSS, National Institute of Health Stroke Scale; MMSE, Mini-Mental State Examination; mRS, modified Rankin Scale; TIA, transient ischemic attack.

The final objective was to describe and compare between treatment arms other characteristics of SAE with available data from CHIMES database in terms of outcome, time to onset and to resolution, and relationship and impact on study treatment.

\section{Statistical Analysis}

Baseline characteristics including risk factors of stroke were tabulated by treatment arm and by occurrence of SAE. Comparison of baseline characteristics between subjects with SAEs and those without SAEs was performed using the chi-square test (or Fisher's exact test if chi-square test was not appropriate) for categorical variables and 2 sample $t$ tests for continuous variables.

Proportions of subjects in the 3 categories of number of SAEs (i.e., no SAE, 1 SAE only, and at least 2 SAEs) were compared between treatment arms using the chi-square test (or Fisher's exact test if chi-square test was not appropriate) in the whole study population and in subsets of subjects with SAEs. The same method was applied to other SAE characteristics such as death and hospitalisa- tion that are expressed as a categorical variable. Relative risk and 95\% CI were provided for binary outcome variables such as subjects with any SAE.

In analysis of individual SAEs with respect to clinical impact, OR was estimated from a logistic regression model using the Generalized Estimating Equations method to account for possible association among multiple SAEs in a subject. Time to resolution of individual SAEs was defined as time from onset of SAE to its resolution or censored at the time of death or end of follow-up if it occurred before resolution. Time to resolution of SAE was analysed using the marginal Cox model approach with a robust sandwich covariance estimate to account for possible association among multiple SAEs in a subject. Hazard ratio from the model was reported with its $95 \%$ CI together with the predicted survival curves. Time to onset of individual SAEs was compared using extended Wilcoxon rank sum test that accounted for possible association of multiple SAEs in a subject as the assumption of proportional hazards was invalid for use of the Cox model. 
Table 2. Distribution of number of SAEs within individual subjects

\begin{tabular}{|c|c|c|c|}
\hline & $\begin{array}{l}\text { MLC601 }(n=542) \\
n / \mathrm{m}(\%)\end{array}$ & $\begin{array}{l}\text { Placebo }(n=545), \\
n / \mathrm{m}(\%)\end{array}$ & $p$ value \\
\hline \multicolumn{3}{|l|}{ In the study population } & 0.002 \\
\hline $\mathrm{nSAE}=0$ & $482 / 542(88.9)$ & $470 / 545(86.2)$ & \\
\hline $\mathrm{nSAE}=1$ & $56 / 542(10.3)$ & $53 / 545(9.7)$ & \\
\hline $\mathrm{nSAE} \geq 2$ & $4 / 542(0.7)$ & $22 / 545(4.0)$ & \\
\hline \multicolumn{3}{|l|}{ In subjects with any $\mathrm{SAE}$} & $<0.001$ \\
\hline $\mathrm{nSAE}=1$ & $56 / 60(93.3)$ & $53 / 75(70.7)$ & \\
\hline $\mathrm{nSAE} \geq 2$ & $4 / 60(6.7)$ & $22 / 75(29.3)$ & \\
\hline \multicolumn{3}{|l|}{ In subjects surviving with any SAE } & 0.006 \\
\hline $\mathrm{nSAE}=1$ & $43 / 47(91.5)$ & $42 / 60(70.0)$ & \\
\hline $\mathrm{nSAE} \geq 2$ & $4 / 47(8.5)$ & $18 / 60(30.0)$ & \\
\hline \multicolumn{3}{|l|}{ In hospitalised subjects surviving with any SAE* } & 0.009 \\
\hline $\mathrm{nSAE}=1$ & $31 / 33(93.9)$ & $33 / 47(70.2)$ & \\
\hline $\mathrm{nSAE} \geq 2$ & $2 / 33(6.1)$ & $14 / 47(29.8)$ & \\
\hline \multicolumn{4}{|c|}{$\begin{array}{l}\text { Numbers in column of each treatment arm are number }(n) \text { and proportion }(\%) \text { of subjects and the correspon- } \\
\text { ding denominator }(\mathrm{m}) \text {. } \\
\text { * Summary includes all SAEs in subjects who were surviving during study follow-up, and had an SAE that } \\
\text { satisfied the SAE criterion of inpatient hospitalisation and/or prolonged hospitalisation. } \\
p \text { values are from chi-square test (or Fisher's exact test if chi-square test is not appropriate). } \\
\text { "Subjects surviving" in the table above means subjects did not die during study follow-up. } \\
\text { SAE, serious adverse event; nSAE, number of SAEs. }\end{array}$} \\
\hline
\end{tabular}

\section{Results}

Of the 1,099 subjects included in CHIMES study, 1,087 were included in the safety analysis with 542 subjects in MLC601 arm and 545 in placebo arm. Twelve subjects who did not receive study treatment were excluded from this as-treated population.

A total of 135 subjects were reported as having had an SAE $($ MLC601 $=60$, placebo $=75)$. As shown in Table 1 , baseline characteristics of the subjects with SAE were rather well balanced within MLC601 and placebo arms, with a trend for higher proportion of subjects with SAEs in MLC601 arm having NIHSS score $>10(p=0.085)$ and a higher NIHSS mean score $(p<0.05)$, and being significantly older in both treatment arms $(p<0.01)$ with a lower MMSE mean score $(p<0.001$ and $p<0.05)$. For cardiovascular risk factors, subjects with SAE compared to those without SAE had higher frequencies of previous history of ischaemic heart disease and smoking in the MLC601 arm ( $p<0.01$ and $p<0.05$ respectively), of diabetes mellitus in placebo arm $(p<0.05)$, and of hyperlipidaemia in both arms $(p<0.01$ and $p<0.001$, respectively).

As evident from our exploratory analysis, distribution of SAE numbers in individual subjects was significantly reduced in MLC601 arm in the overall study population, and in 3 subgroups of subjects with any SAE (all these subjects, those surviving, and those surviving and hospitalised; from $p<0.01$ to $p<0.001$ ). This significant reduction is mostly related to that of subjects with 2 or more SAEs (Table 2).

Among subjects with any AE, the percentage of those having SAE was lower in MLC601 arm compared to placebo one ( 26.1 vs. $34.4 \%$; relative risk 0.76 , $95 \%$ CI 0.57 $1.01 ; p=0.055)$. Analysis of these SAEs' clinical impact (Table 3 ) shows a reduced proportion of hospitalised subjects, significantly for prolonged hospitalisations (1.1 vs. $3.7 \%$; relative risk $0.30,95 \%$ CI $0.12-0.75$; $p<0.01)$. Although not always statistically significant due to the small numbers of events in each subgroup, each individual clinical impact of SAEs was consistently lower in the MLC601 arm in terms of life threatening and IMEs, but not disability. The post hoc count of SAEs classified as IME based on the MedDRA term list was much higher than that recorded by investigators based on their own judgement without reference list. The proportion of subjects with SAEs resulting in at least 2 different impacts was reduced by more than twice in MLC601 arm compared to placebo ( 0.7 vs. $2.0 \% ; p=0.12$ ). The evolution of SAEs was globally non-significantly different between arms, but there was a trend of higher rate of recovery with sequelae $(\sim 35 \%)$ in placebo arm (42.7 vs. $31.7 \% ; p=0.19)$. 
Table 3. Clinical impacts of SAEs

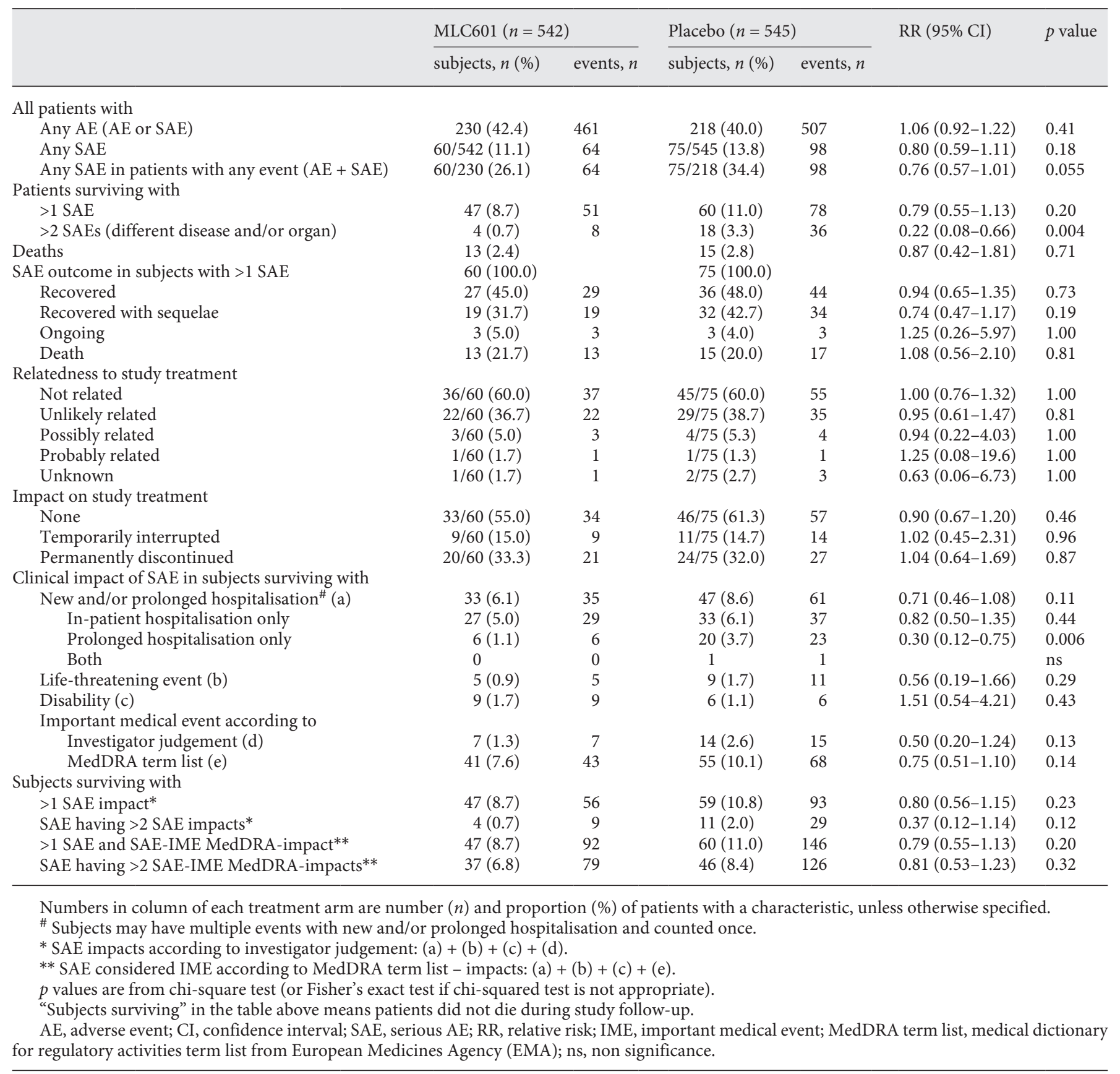

Times to onset and to resolution of SAEs are detailed in online supplemental Table 1 (for all online suppl. material, see www.karger.com/doi/10.1159/000506070). Overall, SAEs appear about 10 days later and resolve significantly quicker in MLC601 arm, for those inducing a prolonged hospitalisation both for individual SAEs by onset ( 7 vs. 20 days; $p=0.03$ ) as shown in Figure 1 and for first SAEs by onset ( 7 vs. 18 days; $p=0.04$ ).

\section{Discussion}

Our exploratory analysis shows that among subjects with SAEs, those receiving MLC601 had fewer SAEs than those in placebo arm. This reduction was observed both in the whole CHIMES study population, all those with any AE or SAE, and those surviving with SAE being hospitalised or not. In addition, most clinical im- 
Fig. 1. Time to resolution of individual serious adverse events (SAE) by onset inducing prolonged hospitalisation in hospitalised subjects surviving with any SAE. Wald test of hazard ratio estimate in the Cox regression model.

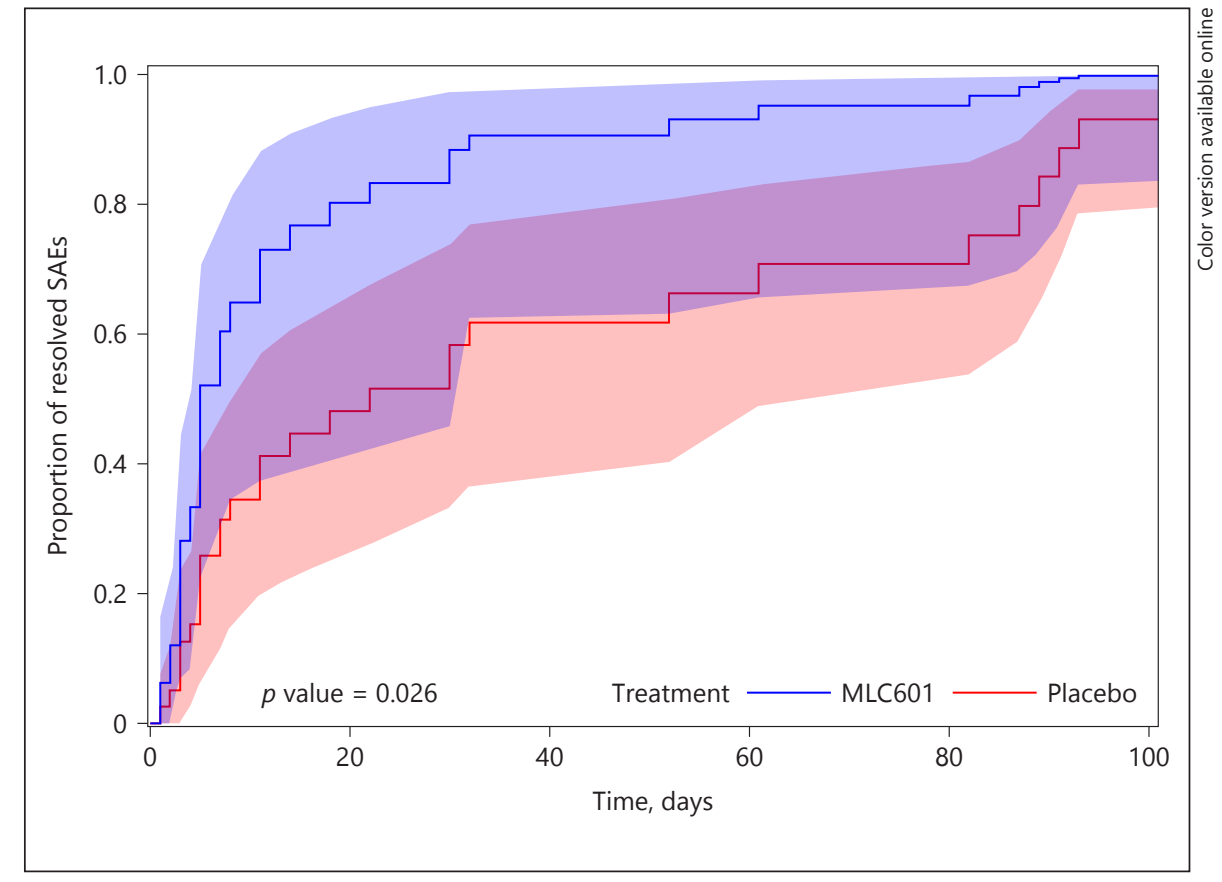

pacts associated with SAEs were reported in fewer subjects receiving MLC601 than placebo. The main clinical impact of SAEs was an increase in hospitalisation time, reduced in MLC601 arm, with the rate of subjects hospitalised for a prolonged period being significantly threefold lower. Our analysis also shows that time to resolution of SAEs is reduced in MLC601 arm both for individual and first SAEs by onset in hospitalised surviving subjects. Concerning SAE outcome, most subjects recovered in both arms, however, with one-third more subjects having recovered with sequelae in placebo arm. To properly assess real treatment benefit, an approach similar to ours recommended using survival time methods accounting for time dependencies and follow-up duration, not only for time-to-event of efficacy endpoints, but also for that of SAEs and their impact, especially in the case of multiple events in single patients, as in our study [12]. Thus, we applied survival time methods in our new analysis of onset and resolution time of SAEs, which clearly indicates a trend for late onset and significantly more rapid resolution of SAEs as illustrated with "survival" curves in hospitalised subjects receiving MLC601.

The main driver for acute care costs is length of stay in hospital $[13,14]$. Studies showed that initial hospitalisation cost for stroke is highly correlated with length of stay, the bulk of cost being attributable to stroke unit stay, increasing with most severe stroke subtypes. The beneficial effects observed in this study of fewer SAEs with quicker and better resolution, and shorter hospitalisation time may reduce the burden on healthcare team and the direct medical costs associated with managing such events in addition to those of standard post-stroke treatments for [15]. Our percentages of subjects affected by SAE impact on hospitalisation might seem low and raise queries about their clinical or economic relevance. However, by applying these reduction rates to millions of stroke survivors each year, this represents hundreds of thousands of SAEs and millions of days of hospitalisation avoided for our patients.

While safety concerns mainly relate to SAEs, less serious but unpleasant side effects are likely to affect quality of life and willingness to continue treatment, which may affect the patient's future [16]. Some prognostic factors may predict some of these complications as it was reported for various post-stroke conditions [17-22]. In our study, more severe stroke, advanced age and the presence of some vascular risk factors increase the risk of SAEs. Appropriately targeted preventative measures would reduce this risk [23]. Interventions to reduce delirium, cardiopulmonary arrest and mortality, drug AEs, infections and falls are helpful.

The CHIMES study has already shown that baseline characteristics such as advanced age, stroke severity, and female gender, portended a poor prognosis for recovery. In these subjects at risk of poor recovery, 
the relative level of recovery was enhanced by MLC601 $[24,25]$. This is consistent with our finding in this study that there were fewer SAEs among subjects receiving MLC601.

Study limitations are as follows. This exploratory analysis was post hoc, and the trial was not originally powered to investigate SAEs. The number was small in some subcategories. Classification of SAEs according to MedDRA term list was done post hoc. Although the study required that all subjects receive standard stroke care, the choice of specific therapies to control medical and social risk factors in each subject was left to the treating physician. Nevertheless, the CHIMES study was a well-monitored, large, double-blinded, randomized trial in which SAEs were hence blindly adjudicated.

\section{Conclusions}

This post hoc and exploratory analysis of SAEs from CHIMES study database shows that subjects receiving a 3-month course of MLC601 experienced less SAEs, with lower rates of harmful clinical impacts, especially in terms of hospitalisation duration. These findings could represent a benefit in terms of reduction of both burden and additional costs. They also suggest the need to invest in further research to better identify patients at risk of SAE and interventions that have a real impact on patient safety.

\section{Disclosure Statement}

C.L.H.C. received grants from the National Medical Research Council of Singapore and funding from the CHIMES Society for conferences and meetings for presentation of the CHIMES study data. Other than CHIMES funding for the trial and its analysis, the other authors and investigators do not have any conflict of interest to declare.

\section{Funding Sources}

The CHIMES-E study was supported by the CHIMES Society, a non-profit organization, and grants were received by CLHC from the National Medical Research Council of Singapore (NMRC/1288/2011 and NMRC/1096/2006).

\section{Appendix}

On behalf of the CHIMES Investigators: Philippines-Jose $\mathrm{C}$ Navarro, Herminigildo H. Gan, Annabelle Lao, Alejandro Baroque II, Johnny Lokin, John Harold B. Hiyadan, Ma. Socorro Sarfati, Randolph John Fangonillo, Neil Ambasing, Carlos Chua, Ma. Cristina San Jose, Joel Advincula, Eli John Berame, Maria Teresa Canete. Singapore-Narayanaswamy Venketasubramanian, Sherry H. Y. Young, Marlie Jane Mamauag, San SanTay, Shrikant Pande, Umapathi Thirugnanam, Rajinder Singh, Hui Meng Chang, Deidre Anne De Silva, Bernard P. L. Chan, Vijay Sharma, Teoh Hock Luen. Thailand- Niphon Poungvarin, Sombat Muengtaweepongsa, Somchai Towanabut, Nijasri Suwanwela, Songkram Chotickanuchit, Siwaporn Chankrachang, Samart Nitinun. Sri Lanka-H. Asita de Silva, Udaya Ranawake, Nirmala Wijekoon. Hong Kong K. S. Lawrence Wong. Malaysia-Gaik Bee Eow.

\section{References}

1 Gurwitz D, McLeod HL. Primum non nocere: adverse drug events must be taken seriously. Pharmacogenomics. 2007 Apr;8(4): 311-4.

2 Chow SC, Pong A. An overview of the regulatory approval process in drug development. Drug Inf J. 1998;32(1_Suppl):1175S-85S.

3 Chuang-Stein C. Safety analysis in controlled clinical trials. Drug Inf J. 1998;32(1_Suppl): 1363S-72S.

4 IQWiG. General Methods, Version 4.2 of 22 April 2015, IQWiG, Cologne, 2015. Available from: https://www.iqwig.de/download/ IQWiG_General_Methods_Version_\%20 4-2.pdf.

5 Talbot GH. Efficacy as an important facet of "safety" in clinical trials: how can we do our best for our patients? Clin Infect Dis. 2008 Dec;47(Suppl 3):S180-5.

6 Phillips R, Hazell L, Sauzet O, Cornelius V. Analysis and reporting of adverse events in randomised controlled trials: a review. BMJ Open. 2019 Mar;9(2):e024537.
7 Wong JW, Churilov L, Dowling R, Mitchell P, Bush S, Kanesan L, et al. Safety of Endovascular Thrombectomy for Acute Ischaemic Stroke in Anticoagulated Patients Ineligible for Intravenous Thrombolysis. Cerebrovasc Dis. 2018;46(5-6):193-9.

8 Chen CL, Young SH, Gan HH, Singh R, Lao AY, Baroque AC 2nd, et al.; CHIMES Study Investigators. Chinese medicine neuroaid efficacy on stroke recovery: a double-blind, placebo-controlled, randomized study. Stroke. 2013 Aug;44(8):2093-100.

9 Chen CL, Venketasubramanian N, Lee CF, Wong KS, Bousser MG, Navarro JC, et al.; CHIMES Study Investigators. Effects of MLC601 on early vascular events in patients after stroke: the CHIMES study. Stroke. 2013 Dec;44(12):3580-3.

10 Venketasubramanian N, Chen CL, Gan RN, Chan BP, Chang HM, Tan SB, et al.; CHIMES Investigators. A double-blind, placebo-controlled, randomized, multicenter study to investigate CHInese Medicine Neuroaid Effi- cacy on Stroke recovery (CHIMES Study). Int J Stroke. 2009 Feb;4(1):54-60.

11 Inspections, Human Medicines Pharmacovigilance \& Committees Division. Important medical event terms list (MedDRA version 22.1). EMA/175234/2019. Available from:https://www.ema.europa.eu/en/documents/other/important-medical-eventterms-list-version-meddra-version-221 en.xls.

12 Bender R, Beckmann L, Lange S. Biometrical issues in the analysis of adverse events within the benefit assessment of drugs. Pharm Stat. $2016 \mathrm{Jul} ; 15(4): 292-6$.

13 Venketasubramanian N, Yin A. Hospital costs for stroke care in Singapore. Cerebrovasc Dis. 2000 Jul-Aug;10(4):320-6.

14 Lugo-Palacios DG, Gannon B, Gittins M, Vail A, Bowen A, Tyson S. Variations in hospital resource use across stroke care teams in England, Wales and Northern Ireland: a retrospective observational study. BMJ Open. 2019 Sep;9(9): e030426. 
15 Hoffman KB, Dimbil M, Kyle RF, Tatonetti NP, Erdman CB, Demakas A, et al. A Drug Safety Rating System Based on Postmarketing Costs Associated with Adverse Events and Patient Outcomes. J Manag Care Spec Pharm. 2015 Dec;21(12):1134-43.

16 Tfelt-Hansen P, Bjarnason NH, Dahlöf C, Derry S, Loder E, Massiou H; Task Force on Adverse Events in Migraine Trials of Subcommittee on Clinical Trial of International Headache Society. Evaluation and registration of adverse events in clinical drug trials in migraine. Cephalalgia. 2008 Jul;28(7): 683-8.

17 Johnston KC, Li JY, Lyden PD, Hanson SK, Feasby TE, Adams RJ, et al.; RANTTAS Investigators. Medical and neurological complications of ischemic stroke: experience from the RANTTAS trial. Stroke. 1998 Feb;29(2):44753.

18 Aslanyan S, Weir CJ, Diener HC, Kaste M, Lees KR; GAIN International Steering Com- mittee and Investigators. Pneumonia and urinary tract infection after acute ischaemic stroke: a tertiary analysis of the GAIN International trial. Eur J Neurol. 2004 Jan;11(1): 49-53.

19 Xu XM, Zou DZ, Shen LY, Liu Y, Zhou XY $\mathrm{Pu}$ JC, et al. Efficacy and feasibility of antidepressant treatment in patients with poststroke depression. Medicine (Baltimore). 2016 Nov;95(45):e5349.

20 Ali M, Sacco RL, Lees KR; VISTA investigators. Primary end-point times, functional outcome and adverse event profile after acute ischaemic stroke. Int J Stroke. 2009 Dec;4(6): $432-42$.

21 Vernino S, Brown RD Jr, Sejvar JJ, Sicks JD, Petty GW, O'Fallon WM. Cause-specific mortality after first cerebral infarction: a population-based study. Stroke. 2003 Aug;34(8): 1828-32.

22 Roth EJ, Lovell L, Harvey RL, Heinemann AW, Semik P, Diaz S. Incidence of and risk factors for medical complications during stroke rehabilitation. Stroke. $2001 \mathrm{Feb} ; 32(2)$ : 523-9.

23 Zegers M, Hesselink G, Geense W, Vincent C, Wollersheim $\mathrm{H}$. Evidence-based interventions to reduce adverse events in hospitals: a systematic review of systematic reviews. BMJ Open. 2016 Sep;6(9):e012555.

24 Chankrachang S, Navarro JC, de Silva DA, Towanabut S, Chua CL, Lee CF, et al.; CHIMES Study Investigators. Prognostic factors and treatment effect in the CHIMES study. J Stroke Cerebrovasc Dis. 2015 Apr; 24(4):823-7.

25 Venketasubramanian N, Lee CF, Young SH, Tay SS, Umapathi T, Lao AY, et al.; CHIMESE Study Investigators. Prognostic Factors and Pattern of Long-Term Recovery with MLC601 (NeuroAiD ${ }^{\mathrm{TM}}$ ) in the Chinese Medicine NeuroAiD Efficacy on Stroke Recovery - Extension Study. Cerebrovasc Dis. 2017;43(1-2): 36-42. 